\title{
Isoliquiritigenin inhibits cell proliferation and migration through the PI3K/AKT signaling pathway in A549 lung cancer cells
}

\author{
TAO TIAN ${ }^{1}$, JINPENG SUN $^{2}$, JIANXIN WANG ${ }^{1}$, YANCHUN LIU $^{1}$ and HAITAO LIU ${ }^{1}$ \\ ${ }^{1}$ Department of Respiratory Medicine, Hebei Medical University Affiliated North China Petroleum Bureau General Hospital, \\ Renqiu, Hebei 062552; ${ }^{2}$ Department of Surgical Oncology, Cangzhou Hospital of Integrated Traditional Chinese and \\ Western of Hebei Province, Cangzhou, Hebei 061001, P.R. China
}

Received November 26, 2017; Accepted July 13, 2018

DOI: $10.3892 / \mathrm{ol} .2018 .9344$

\begin{abstract}
The present study aimed to investigate the molecular mechanisms of inhibition of Isoliquiritigenin (ISL) on the proliferation and migration of A549 cells. A549 cells were cultured in vitro, and the effects of ISL inhibition were examined using cell counting kit- 8 , Transwell invasion and flow cytometric assays. Western blot analysis was also performed to detect cell apoptosis and the expression of phosphatidylinositol 3-kinase (PI3K)/AKT serine/threonine kinase (AKT) signaling pathway-associated proteins. The results demonstrated a significant inhibition of proliferation and migration of A549 cells when treated with ISL $(\mathrm{P}<0.05)$. Furthermore, ISL treatment significantly downregulated the expression of E-cadherin, and upregulated the expression of $\mathrm{N}$-cadherin and vimentin. Flow cytometric analysis revealed a significant increase in cell apoptosis in the ISL group as well as the expression of pro-apoptotic proteins Bcl-2-associated $\mathrm{X}$ protein and active caspase-3. Conversely, the expression of anti-apoptotic protein B-cell lymphoma 2 was decreased. There was a significant decrease in the phosphorylation of AKT and mammalian target of rapamycin, and in the expression of cell proliferation proteins P70 and cyclin D1 in ISL-treated cells. In conclusion, ISL has significant inhibitory effects on the proliferation and migration of A549 cells by promoting cell apoptosis. The mechanism may involve of PI3K/AKT signaling pathways in A549 cells, which may a potential therapeutic target for the treatment of lung cancer.
\end{abstract}

\section{Introduction}

Lung cancer is one of the most common primary malignant tumor types in numerous countries, including China and North

Correspondence to: Dr Haitao Liu, Department of Respiratory Medicine, Hebei Medical University Affiliated North China Petroleum Bureau General Hospital, 17 Huizhan Street, Renqiu, Hebei 062552, P.R. China

E-mail: liuhtdoctor2012@163.com

Key words: isoliquiritigenin, lung cancer, proliferation, migration, PI3K/AKT pathway
America (1). There are two primary types of lung cancer: Small-cell lung cancer (SCLC) and non-small-cell lung cancer (NSCLC) (1). NSCLC accounts for 75-85\% of lung cancer cases $(2,3)$. Compared with SCLC, NSCLC cells grow and divide more rapidly, resulting in diffusion and metastasis at an earlier stage of disease (4). However, only $15 \%$ of patients with NSCLC are diagnosed at an early stage of the disease and current treatment modalities, including surgical resection and chemoradiation are inadequate (5). Even if diagnosis occurs at stage I (6), the 10-year survival rate for patients with NSCLC is $\sim 50 \%(7,8)$. The 5-year survival rate of patients with NSCLC is only $16 \%$ due to early tumor metastasis and recurrence $(9,10)$. Therefore, the development of novel diagnostic strategies and natural antineoplastic drugs for the treatment of lung cancer have become the focus of clinical research.

Isoliquiritigenin (2'-4'-4-trihydroxychalcone, ISL), a flavonoid identified in licorice, and is a potent antioxidant with anti-inflammatory, antioxidant and antitumor capabilities (11-13). ISL exhibits an inhibitory effect on anti-proliferative activities in gastric cancer, prostate cancer, hepatocellular carcinoma (HCC), breast cancer, melanoma, and lung cancer cells (14-19). Furthermore, ISL has been demonstrated to block the proliferation rate of HCC cells in a dose- and time-dependent manner (20), and induce apoptosis and autophagy in various cancer cells, including drug-resistant breast cancer MCF-7/ADR cells, and endometrial cancer Ishikawa and HEC-1A cells $(21,22)$. In recent years, the anticancer mechanism of ISL has been thoroughly studied. In endometrial cancer cells, ISL was demonstrated to induce cell cycle arrest in the sub-G1 or $\mathrm{G} 2 / \mathrm{M}$ phase through the $\mathrm{p} 53 / \mathrm{p} 21$ pathway, and promote cell apoptosis and autophagy via activation of the extracellular signal-regulated kinase pathway (22). In addition, previous studies have demonstrated that the phosphatidylinositol 3-kinase (PI3K)/AKT serine/threonine kinase (AKT) signaling pathway is also involved in ISL-induced cell apoptosis and proliferation $(23,24)$; however, the possible regulation of the PI3K/AKT signaling pathway and its downstream pathways by ISL in the context of lung cancer has received relatively little attention.

In the present study, the inhibitory effects of ISL on cell proliferation, migration, invasion and apoptosis in A549 lung cancer cells were examined. Furthermore, in order to establish 
the anticancer mechanism of ISL, the expression of B-cell lymphoma 2 (Bcl-2), Bcl-2-associated X protein (BAX), active caspase-3, Cyclin D1, P70, AKT, phosphorylated (p)-AKT, mammalian target of rapamycin (mTOR) and p-mTOR were assayed, due to their association with cell apoptosis and the PI3K/AKT signaling pathway.

\section{Materials and methods}

Cell culture and treatment. A549 cells were obtained from the Type Culture Collection of the Chinese Academy of Sciences (Shanghai, China). Cells were maintained in a monolayer culture with $5 \% \mathrm{CO}_{2}$ at $37^{\circ} \mathrm{C}$ in RPMI-1640 (HyClone; GE Healthcare Life Sciences, Logan, UT, USA) supplemented with $10 \%$ fetal bovine serum (FBS; Gibco; Thermo Fisher Scientific, Inc., Waltham, MA, USA), $10 \mathrm{U} / \mathrm{ml}$ penicillin (Sigma-Aldrich Merck KGaA, Darmstadt, Germany) and $100 \mu \mathrm{g} / \mathrm{ml}$ streptomycin (Sigma-Aldrich; Merck KGaA). The cells in the logarithmic phase were washed with PBS and detached with Trypsin-EDTA (cat. no. 25200056; Thermo Fisher Scientific, Inc.,) for $3 \mathrm{~min}$ at $37^{\circ} \mathrm{C}$. Confluent A549 cells were then seeded into 6-well plates at a density of $5 \times 10^{4}$ cells/well with the RPMI-1640 medium for subsequent experiments. ISL (MedChem Express, Monmouth Junction, NJ, USA) was dissolved in DMSO (Amresco, LLC, Solon, OH, USA) as the stock solution and final concentrations of the compounds tested were prepared by diluting the stock solution with RPMI-1640. A549 cells were treated with ISL $(20 \mu \mathrm{M})$ for $24 \mathrm{~h}$, and the cells treated with $0.1 \%$ DMSO were designated the negative control group (NC).

Cell proliferation assay. Inhibition of cell proliferation by ISL was measured using the cell counting kit-8 (CCK8) assay. Cells were plated in $96-$ well plates $\left(1 \times 10^{3}\right.$ cells/well). Following a $24 \mathrm{~h}$ incubation at $37^{\circ} \mathrm{C}$, cells of the experimental group were treated with ISL $(20 \mu \mathrm{M})$ for 24,48 and $72 \mathrm{~h}$, and the negative control group was treated with $0.1 \%$ DMSO. CCK8 (Beijing Solarbio Science \& Technology Co., Ltd., Beijing, China) test solution (10 $\mu \mathrm{l})$ was added to each well. Following a $90 \mathrm{~min}$ incubation, the absorbance was measured on an ELISA reader at a wavelength of $450 \mathrm{~nm}$. A curve was drawn for proliferation inhibition analysis. The experiment was repeated in triplicate.

Cell migration and invasion assay. For the invasion assay, a Transwell chamber (EMD Millipore) was used to structure the cell invasion model. In summary, $100 \mu \mathrm{l}$ of Matrigel (BD Biosciences, San Jose, CA, USA), melted overnight and diluted with serum-free RPMI-1640 medium in a 1:6 ratio, was added to the upper chamber of 24-well plates, following incubation for $4-6 \mathrm{~h}$ at $37^{\circ} \mathrm{C}$ with $5 \% \mathrm{CO}_{2}$. The medium was removed, $500 \mu \mathrm{l}$ of serum-free medium was added to the lower chamber for $30 \mathrm{~min}$ at $37^{\circ} \mathrm{C}$ to hydrate the basement membrane. A549 cells $\left(1 \times 10^{5}\right)$ treated with ISL $(20 \mu \mathrm{M})$ or $0.1 \%$ DMSO for $48 \mathrm{~h}$ at $37^{\circ} \mathrm{C}$ were seeded in the upper chamber in $100 \mu \mathrm{l}$ of serum-free medium. The lower chamber was filled with $500 \mu 1$ of medium supplemented with $10 \%$ FBS. Following a $24 \mathrm{~h}$ incubation at $37^{\circ} \mathrm{C}$, non-invasive cells on the upper surface of the Matrigel were gently scrubbed with a cotton swab and migrated cells on the lower surface were washed with PBS, fixed with $4 \%$ paraformaldehyde for $30 \mathrm{~min}$ at room temperature, stained with $0.1 \%$ crystal violet for $20 \mathrm{~min}$ at room temperature and recorded for images under a light microscope (Olympus Corporation, Tokyo, Japan; magnification, magnification, x100). For the migration assay, the same method as the invasion assay was used, only without Matrigel. Each assay was performed in triplicate.

Analysis of cell apoptosis. Apoptosis was assessed by flow cytometry analysis, in which the percentage of apoptotic cells was determined using an Annexin V-fluorescein isothiocyanate (FITC) apoptosis detection kit (Beijing 4A Biotech, Beijing, Co., Ltd., Beijing, China), according to the manufacturer's protocol. The cells either treated with ISL $(20 \mu \mathrm{M})$ or $0.1 \%$ DMSO for $48 \mathrm{~h}$ at $37^{\circ} \mathrm{C}$ were cultured with serum-free medium for $24 \mathrm{~h}$ at $37^{\circ} \mathrm{C}$. The cells were subsequently collected and washed twice with chilled PBS for 5 min at $0^{\circ} \mathrm{C}$, resuspended in binding buffer $\left(1 \times 10^{6}\right.$ cells/well $)$ and incubated with FITC-conjugated Annexin V (5 $\mu$ l) in the dark for $30 \mathrm{~min}$ at room temperature. Following the addition of $10 \mu 1$ of propidium iodide (PI) and $400 \mu 1$ of PBS for $5 \mathrm{~min}$ at room temperature, the cells were analyzed using a FACS caliber instrument. FlowJo software (version 7.6.5; FlowJo LLC, Ashland, OR, USA) was used to analyze the flow cytometry results. The experiment was performed in triplicate.

Protein extraction and western blot analysis. Protein expression was detected using a western blot assay. The A549 cells treated with ISL $(20 \mu \mathrm{M})$ or $0.1 \%$ DMSO for $48 \mathrm{~h}$ in 6-well plates were harvested and lysed in RIPA buffer (CWBio, Inc., Beijing, China) at $4^{\circ} \mathrm{C}$. Proteins were extracted and the concentrations were measured using the bicinchoninic acid method. A total of $20 \mu \mathrm{g}$ of the total proteins were fractionated electrophoretically by $10 \%$ SDS-PAGE gels (Beyotime Institute of Biotechnology, Beijing, China) and transferred onto polyvinylidene fluoride membranes (EMD Millipore, Billerica, MA, USA), which were then blocked with $5 \%$ nonfat milk for $1 \mathrm{~h}$ at room temperature, and then cultured overnight at $4^{\circ} \mathrm{C}$ with the following primary antibodies: E-cadherin (dilution, 1:1,000; cat. no. 20874-1-AP; rabbit polyclonal), N-cadherin (dilution, 1:1,000; cat. no. 22018-1-AP; rabbit polyclonal), vimentin (dilution, 1:1,000; cat. no. 10366-1-AP; rabbit polyclonal), Bcl-2 (dilution, 1:1,000; cat. no. 12789-1-AP; rabbit polyclonal), Bax (dilution, 1:1,000; cat. no. 50599-2-lg; rabbit polyclonal), active caspase-3 (dilution, 1:1,000; cat. no. 19677-1-AP; rabbit polyclonal), cyclin D1 (dilution, 1:1,000; cat. no. 60186-1-lg; mouse monoclonal), P70 (dilution, 1:1,000; cat. no. 14485-1-AP; rabbit polyclonal) and $\beta$-tubulin (dilution, 1:5,000; cat. no. 10094-1-AP; rabbit polyclonal; all from ProteinTech Group, Inc. Chicago, IL, USA), AKT (dilution, 1:1,000; cat. no. 4691; rabbit polyclonal), p-AKT (dilution, 1:1,000; cat. no. 4060; rabbit polyclonal), mTOR (dilution, 1:1,000; cat. no. 2983; rabbit polyclonal) and p-mTOR (dilution, 1:1,000; cat. no. 5536; rabbit polyclonal) (all from Cell Signaling Technology,Inc., Danvers, MA, USA). $\beta$-tubulin was used as a loading control. The membranes were washed with Tris-buffered saline with $0.1 \%$ Tween-20 in triplicate and incubated with horseradish peroxidase conjugated goat anti-rabbit or goat anti-mouse IgG secondary antibodies 
A

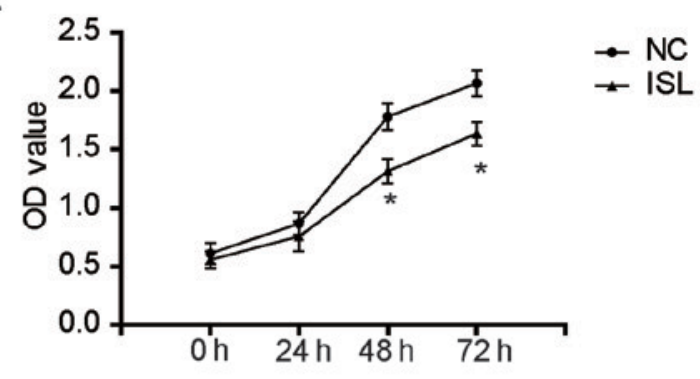

B
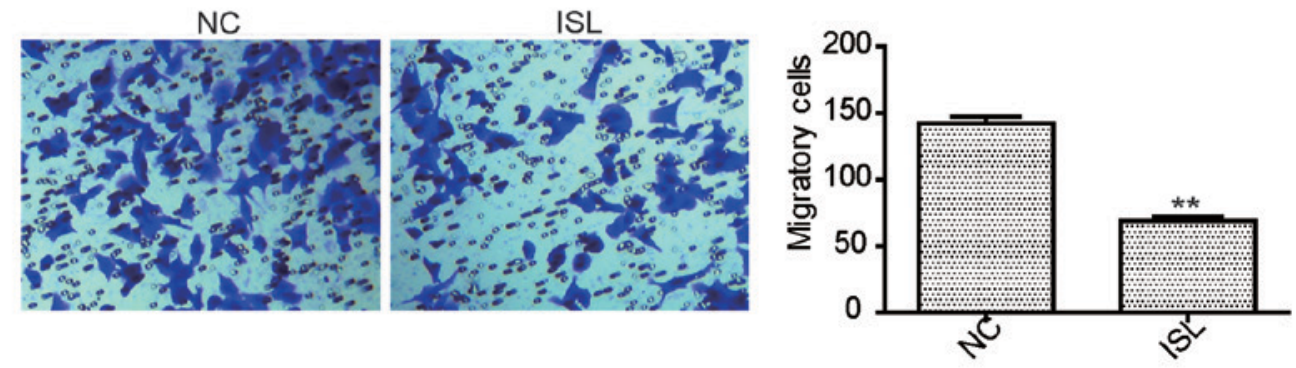

C
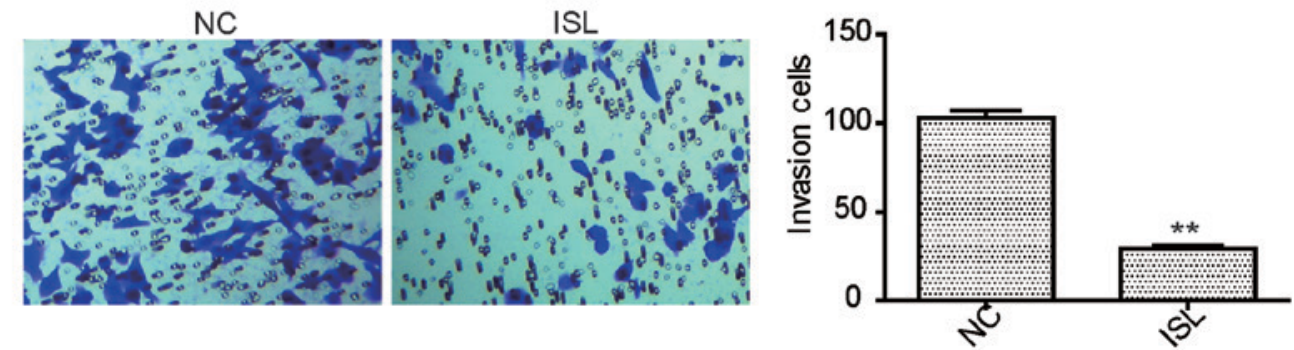

D
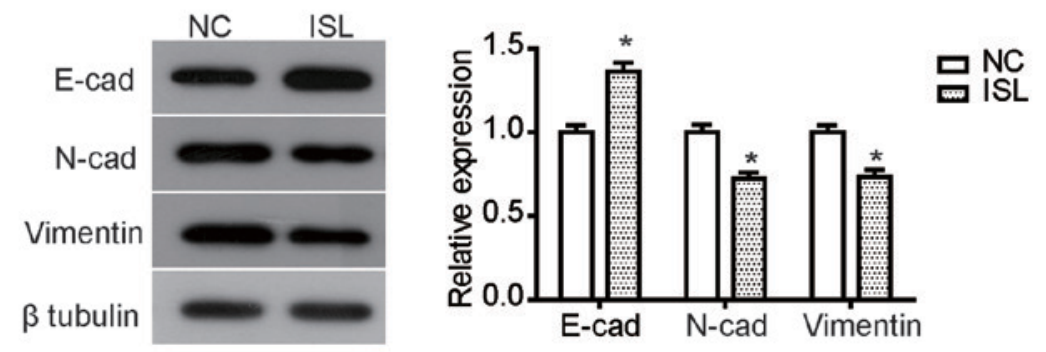

Figure 1. ISL treatment inhibits proliferation, migration, and invasion of A549 cells. (A) The inhibition in proliferation of A549 cells treated with ISL (20 $\mu \mathrm{M})$ following a 24,48 and $72 \mathrm{~h}$ incubation. (B) ISL treatment $(20 \mu \mathrm{M})$ for $48 \mathrm{~h}$ inhibited the migration of A549 cells compared with cells treated with DMSO (optical microscope; magnification, x100). (C) ISL treatment $(20 \mu \mathrm{M})$ for $48 \mathrm{~h}$ inhibited the invasion of A549 cells compared with the negative control group (optical microscope; magnification, x100). (D) Western blot analysis of the expression of E-cadherin, N-cadherin and vimentin in A549 cells following treatment with ISL for $48 \mathrm{~h}$. The data was calculated using mean \pm standard deviation of three repeats. ${ }^{*} \mathrm{P}<0.05$ and ${ }^{* *} \mathrm{P}<0.01$ compared with NC. OD, optical density; NC, negative control; ISL, isoliquiritigenin.

(cat. nos. SA00001-2 and SA00001-1, respectively; dilution, 1:5,000; ProteinTech Group, Inc.) for $1 \mathrm{~h}$ at room temperature. Chemiluminescence detection was performed using a standard enhanced chemiluminescence kit (cat. no. W0049M; CWBio, Inc.), according to the manufacturer's protocol. The gray values were obtained by Quantity One v4.6.2 software (BioRad, Inc., California, USA) for calculating the protein relative content. The experiment was repeated three times independently.

Statistical analysis. The data were analyzed with the software SPSS 17.0 (SPSS Inc., Chicago, IL, USA). All values are expressed as the mean \pm standard deviation. Statistical comparisons between two groups were made using an independent sample two-tailed Student's t-test. $\mathrm{P}<0.05$ was considered to indicate a statistically significant difference.

\section{Results}

Inhibition of ISL on the proliferation of A549 cells. The anti-proliferative effect of ISL on the lung cancer A549 cells was examined using CCK8. Following exposure to ISL $(20 \mu \mathrm{M})$ for $24-72 \mathrm{~h}$, cell viability of A549 cells was assessed. As illustrated in Fig. 1A, the proliferation of A549 cells treated with ISL for $48 \mathrm{~h}$ or $72 \mathrm{~h}$ was significantly suppressed compared with the $\mathrm{NC}$ group $(\mathrm{P}<0.05)$. 

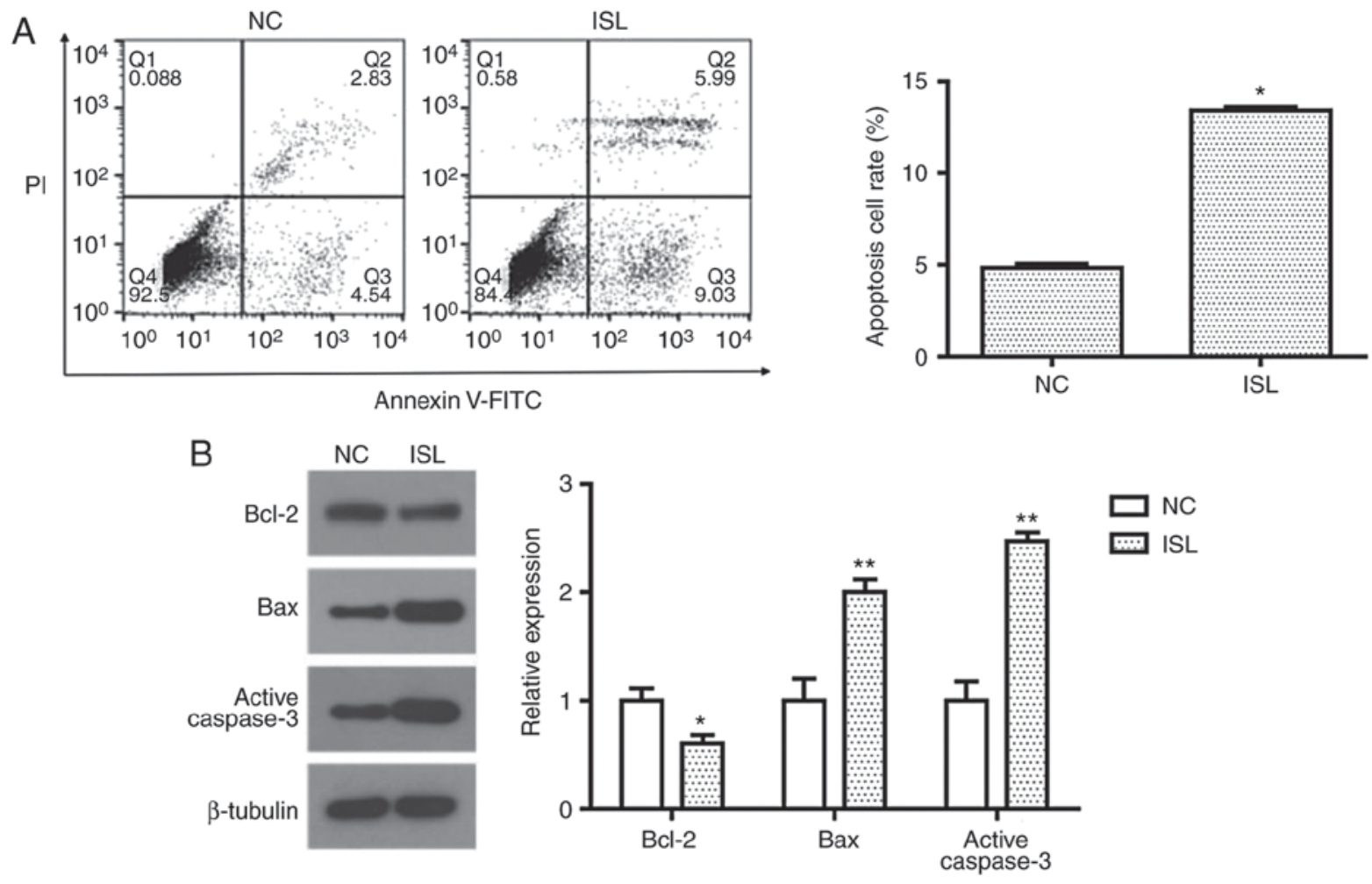

Figure 2. ISL induces apoptosis of A549 cells via the mitochondrial apoptosis pathway. (A) Flow cytometric analysis of ISL-treated A549 cells demonstrated that $20 \mu \mathrm{M}$ ISL treatment for $48 \mathrm{~h}$ promoted the apoptosis of A549 cells. (B) Expression of Bcl-2, Bax and active caspase-3 in A549 cells following ISL treatment was determined using western blot analysis. $\beta$-tubulin was used as the loading control. The data was calculated using mean \pm standard deviation of three repeats. ${ }^{*} \mathrm{P}<0.05$ and ${ }^{* *} \mathrm{P}<0.01$ compared with NC. NC, negative control; ISL, Isoliquiritigenin; Bax, Bcl-2-associated X; Bcl-2, B-cell lymphoma 2.

Inhibition of ISL on the migration and invasion of A549 cells. To assay the inhibition of ISL on the migration and invasion of A549 cells, a Transwell invasion experiment was performed. Migration and invasion were assessed following treatment ISL $(20 \mu \mathrm{M})$ for $24 \mathrm{~h}$. The results demonstrated that the migration of A549 cells treated with ISL was effectively inhibited compared with the NC (Fig. 1B). Furthermore, ISL treatment also significantly suppressed the invasion ability of A549 cells (Fig. 1C). To further explore the relevant mechanism of inhibition on cell mobility caused by ISL, the expression of cell metastasis-associated proteins was examined. As illustrated in Fig. 1D, ISL treatment led to a significant increase in the expression of E-cadherin, but reduced the expression of $\mathrm{N}$-cadherin and vimentin, suggesting that the inhibition of migration and invasion by ISL may be mediated by regulating the expression of these proteins.

ISL induces apoptosis in A549 cells. Cell apoptosis was evaluated by determining the percentage of A549 cells undergoing apoptotic cell death following treatment with ISL $(20 \mu \mathrm{M})$ with Annexin V-FITC/PI staining. Flow cytometric analysis demonstrated that ISL $(20 \mu \mathrm{M})$ treatment significantly promoted apoptosis in A549 cells compared with the NC (Fig. 2A). To investigate the apoptotic pathway induced by ISL, the expression levels of Bcl-2, Bax and active caspase-3 were detected by western blot analysis. When compared with the control group, the expression of anti-apoptosis protein $\mathrm{Bcl}-2$ was significantly decreased, while pro-apoptosis proteins Bax and active caspase- 3 were significantly increased following treatment with ISL (Fig. 2B). These results suggest that the induction of apoptosis in ISL-treated A549 cells may be associated with the mitochondrial apoptosis pathway.

ISL inhibits the activation of the PI3K/AKT signaling pathway. The PI3K/AKT signaling pathway is important in the occurrence and development of cancer, as Akt and mTOR proteins serve essential role in the proliferation and migration of tumor cells (25). In the present study the expression levels of these proteins were assessed by western blot analysis. As illustrated in Fig. 3, the phosphorylation levels of Akt and mTOR in the ISL-treated A549 cells were significantly decreased, and the expression levels of their downstream proteins P70 and Cyclin D1 were decreased accordingly.

\section{Discussion}

ISL belongs to the family of hydroxy chalcone compounds and has a flavonoid composition extracted from licorice roots (15). Results from several studies indicate that ISL has antitumor, anti-virus and anti-inflammatory effects, with treatment resulting in increased vascular elasticity, inhibition of lipid peroxidation and numerous biological effects, including proliferation, apoptosis and autophagy (14-19). Its antitumor effects have attracted much attention in recent years. A previous study revealed that the active extract containing liquiritin, isoliquiritin and ISL may inhibit cell proliferation, and induce cell cycle arrest and apoptosis of A549 cells (26). However, it is unclear which molecule in the active extract is responsible for causing this anti-cancer effect. Therefore, 
A

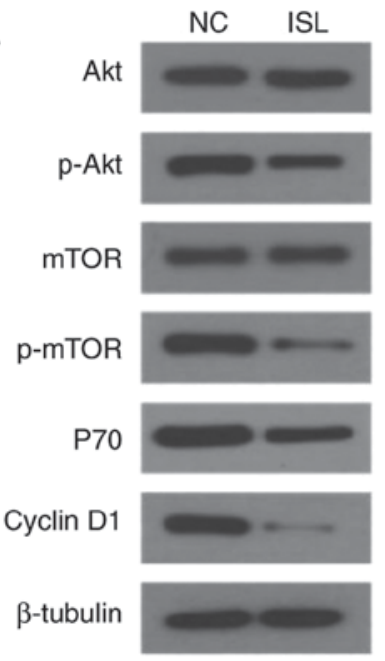

B
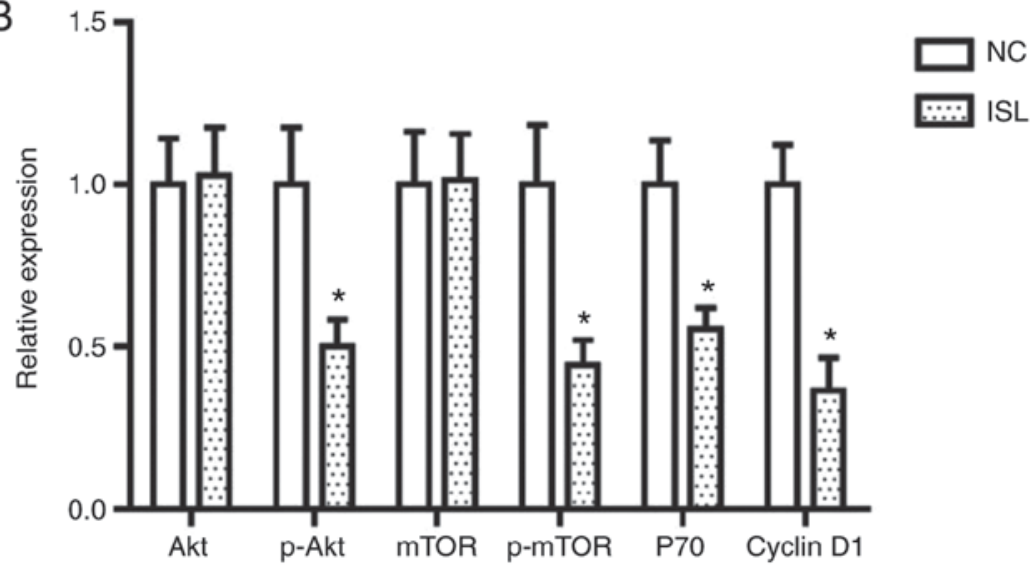

Figure 3. ISL treatment inhibits the activation of the PI3K/AKT signaling pathway in A549 cells. (A) Effects of ISL on protein expression of AKT, mTOR, p-AKT, p-mTOR, P70 and Cyclin D1 in A549 cells, determined using western blot analysis. $\beta$-tubulin was used as the loading control. (B) Quantitative results of protein expression. The data was calculated using mean \pm standard deviation of three repeats. * $\mathrm{P}<0.05$ compared with $\mathrm{NC}$. NC, negative control; ISL, Isoliquiritigenin; PI3K, phosphatidylinositol 3-kinase; AKT, AKT serine/threonine kinase; mTOR, mammalian target of rapamycin; p, phosphorylated.

the present study further investigated the effect of ISL on the biological behaviors of NSCLC cells. In the present study, ISL was demonstrated to significantly inhibit the proliferation, migration and invasion of A549 cells, and induce cell apoptosis, suggesting that ISL may have an anti-cancer effect on the growth and metastasis of NSCLC cells. In addition, ISL significantly upregulated the expression of E-cadherin, and downregulated the expression of $\mathrm{N}$-cadherin and vimentin in A549 cells. E-cadherin is a marker expressed on epithelial cells, and a decrease in expression of E-cadherin is one of the primary features of epithelial-to-mesenchymal transition (EMT), which is a pivotal mechanism involved in modulation of cell migration and invasion. Furthermore, $\mathrm{N}$-cadherin and vimentin are mesenchymal markers involved in EMT process (27). Thus, the results of the present study suggest that ISL suppresses migration and invasion of A549 cells via inhibition of the EMT process. Cell apoptosis, a tightly regulated process of cell death, is associated to growth, organized stability, tumors, and autoimmune and neurodegenerative disease $(28,29)$. Since Kerr established this concept in 1972 (30), the phenomenon of apoptosis has been extensively researched. Cell apoptosis is currently one of the most popular fields in life science research; however, the molecular and biochemical mechanisms of apoptosis have yet to be completely elucidated. The Bcl-2 and caspase protein families are of the most well-studied among the multitude of apoptosis-regulating genes (31). The Bcl-2 family has two categories: Apoptosis inhibition and apoptosis promotion (32). The Bcl-2 family proteins are important regulatory factors in response to apoptosis, and Bcl-2/Bax are well-established as the most important pair of function contradictory regulate genes in the process of apoptosis regulation (33). Caspase-3 is an important apoptosis execution protease, and the association between Bcl-2, Bax and caspase- 3 has become a principal focus in the study of apoptosis (34). In the present study, the contribution of Bcl-2, Bax and active caspase-3 proteins to ISL-induced cell apoptosis was examined. The results demonstrated that
ISL significantly induced cell apoptosis in A549 cells via the mitochondrial apoptosis pathway. The anticancer role of ISL in the progression of NSCLC will be investigated in animal models in further studies.

The anti-cancer mechanism of ISL has also been widely studied in recent years. ISL may cause glioma U87 cells to enter the $S$ phase and G2/M checkpoint of the cell cycle by increasing P21/cyclin dependent kinase inhibitor 1A and P27 proteins (35). In prostate cancer, ISL was demonstrated to downregulate the proliferation of DU145 cells through inhibition of AKT phosphorylation, ERB and PI3K/AKT signaling pathways (36). Kang et al (37) demonstrated that ISL inhibits the formation of new blood vessels through blocking of the c-Jun N-terminal kinase and p38/MAPK signaling pathways, subsequently inhibiting the activation of matrix metalloproteinases. The PI3K/AKT signaling pathway and its downstream pathways have been demonstrated to serve essential roles in growth regulation of lung cancer. For example, docosahexaenoic acid induces cell death in human NSCLC cells by repressing mTOR via PI3K/AKT inhibition (38). Plumbagin induces apoptotic and autophagic cell death through inhibition of the PI3K/Akt/mTOR pathway in human NSCLC cells (39). However, the contribution of the PI3K/AKT signaling pathway to the anticancer effects of ISL in lung cancer has received relatively little attention. The present study demonstrated that ISL significantly reduced the expression of p-AKT, p-mTOR, and the downstream proteins P70 and Cyclin D1, suggesting that ISL inhibits the activation of the PI3K/AKT signaling pathway in A549 cells. Taken together, ISL induces growth inhibition and apoptosis through inhibiting the activation of the PI3K/AKT/mTOR signaling pathway in A549 lung cancer cells. It is suggested that the PI3K/AKT/mTOR signaling pathway serves an important role in IS -mediated A549 cell apoptosis.

In conclusion, the results of the present study demonstrate that ISL inhibits the proliferation, migration and invasion of A549 lung cancer cells and induces cell apoptosis, which may be associated with the downregulation of the PI3K/AKT/mTOR 
signaling pathway proteins, and the downstream proteins P70 and CyclinD1. And further studies should investigate ISL as a therapeutic agent for inhibiting the growth and metastasis of NSCLC.

\section{Acknowledgements}

Not applicable.

\section{Funding}

Not applicable.

\section{Availability of data and materials}

All data generated or analyzed in the study are included in the present published article.

\section{Authors' contributions}

TT and HL conceived and designed the study. The experiments in the present study were performed by TT, JS and JW. The statistical analysis was performed by YL and HL, and HL has written the manuscript. All authors have read and approved this manuscript.

\section{Ethics approval and consent to participate}

The present study was approved by the Research Ethics Committee of Hebei Medical University Affiliated North China Petroleum Bureau General Hospital.

\section{Patient consent for publication}

Not applicable.

\section{Competing interests}

The authors declare that they have no competing interests.

\section{References}

1. Hoffman PC, Mauer AM and Vokes EE: Lung cancer. Lancet 355 479-485, 2000

2. Bilello KS, Murin S and Matthay RA: Epidemiology, etiology, and prevention of lung cancer. Clin Chest Med 23: 1-25, 2002.

3. Sundar R, Soong R, Cho BC, Brahmer JR and Soo RA: Immunotherapy in the treatment of non-small cell lung cancer. Lung Cancer 85: 101-109, 2014.

4. Zhao J, Qiao CR, Ding Z, Sheng YL, Li XN, Yang Y, Zhu DY, Zhang CY, Liu DL, Wu K and Zhao S: A novel pathway in NSCLC cells: miR-191, targeting NFIA, is induced by chronic hypoxia, and promotes cell proliferation and migration. Mol Med Rep 15: 1319-1325, 2017.

5. Centers for Disease Control and Prevention (CDC): Recent trends in mortality rates for four major cancers, by sex and race/ethnicity-united states, 1990-1998. MMWR Morb Mortal Wkly Rep 51: 49-53, 2002.

6. Scott WJ, Howington J, Feigenberg S, Movsas B, Pisters K and American College of Chest Physicians: Treatment of non-small cell lung cancer stage I and stage II: ACCP evidence-based clinical practice guidelines (2nd edition). Chest 132 (Suppl 3): S234-S242, 2007.

7. Rosell R and Karachaliou N: Lung cancer: Maintenance therapy and precision medicine in NSCLC. Nat Rev Clin Oncol 10: $549-550,2013$
8. Sun SJ, Lin Q, Ma JX, Shi WW, Yang B and Li F: Long non-coding RNA NEAT1 acts as oncogene in NSCLC by regulating the Wnt signaling pathway. Eur Rev Med Pharmacol Sci 21: 504-510, 2017.

9. Miller KD, Siegel RL, Lin CC, Mariotto AB, Kramer JL, Rowland JH, Stein KD, Alteri R and Jemal A: Cancer treatment and survivorship statistics, 2016. Ca Cancer J Clin 66: 271-289, 2016.

10. Zhang Y, Li ZY, Hou XX, Wang X, Luo YH, Ying YP and Chen G: Clinical significance and effect of AEG-1 on the proliferation, invasion, and migration of NSCLC: A study based on immunohistochemistry, TCGA, bioinformatics, in vitro and in vivo verification. Oncotarget 8: 16531-16552, 2017.

11. Vaya J,Belinky PA and Aviram M: Antioxidant constituents from licorice roots: Isolation, structure elucidation and antioxidative capacity toward LDL oxidation. Free Radic Biol Med 23: 302-313, 1997.

12. Chan SC, Chang YS, Wang JP, Chen SC and Kuo SC: Three new flavonoids and antiallergic, anti-inflammatory constituents from the heartwood of dalbergia odorifera. Planta Med 64: 153-158, 1998.

13. Yamamoto S, Aizu E, Jiang H, Nakadate T, Kiyoto I, Wang JC and Kato R: The potent anti-tumor-promoting agent isoliquiritigenin. Carcinogenesis 12: 317-323, 1991.

14. Ma J, Fu NY, Pang DB, Wu WY and Xu AL: Apoptosis induced by isoliquiritigenin in human gastric cancer MGC-803 cells. Planta Med 67: 754-757, 2001.

15. Kanazawa M, Satomi Y, Mizutani Y, Ukimura O, Kawauchi A, Sakai T, Baba M, Okuyama T, Nishino H and Miki T: Isoliquiritigenin inhibits the growth of prostate cancer. Eur Urol 43: 580-586, 2003.

16. Hsu YL, Kuo PL, Lin LT and Lin CC: Isoliquiritigenin inhibits cell proliferation and induces apoptosis in human hepatoma cells. Planta Med 71: 130-134, 2005.

17. Wang KL, Hsia SM, Chan CJ, Chang FY, Huang CY, Bau DT and Wang PS: Inhibitory effects of isoliquiritigenin on the migration and invasion of human breast cancer cells. Expert Opin Ther Targets 17: 337-349, 2013

18. Chen XY, Li DF, Han JC, Wang B, Dong ZP, Yu LN, Pan ZH, Qu CJ, Chen Y, Sun SG and Zheng QS: Reprogramming induced by isoliquiritigenin diminishes melanoma cachexia through mTORC2-AKT-GSK3 $\beta$ signaling. Oncotarget 8: 34565-34575, 2017.

19. Hsu YL, Kuo PL, Chiang LC and Lin CC: Isoliquiritigenin inhibits the proliferation and induces the apoptosis of human non-small cell lung cancer a549 cells. Clin Exp Pharmacol Physiol 31: 414-418, 2004.

20. Cao LJ, Li HD, Yan M, Li ZH, Gong H, Jiang P, Deng Y, Fang PF and Zhang BK: The protective effects of isoliquiritigenin and glycyrrhetinic acid against triptolide-induced oxidative stress in hepG2 cells involve Nrf2 activation. Evid Based Complement Alternat Med 2016: 8912184, 2016.

21. Wang Z, Wang N, Liu P, Chen Q, Situ H, Xie T, Zhang J, Peng $C$, Lin $Y$ and Chen J: MicroRNA-25 regulates chemoresistance-associated autophagy in breast cancer cells, a process modulated by the natural autophagy inducer isoliquiritigenin. Oncotarget 5: 7013-7026, 2014.

22. Wu CH, Chen HY, Wang CW, Shieh TM, Huang TC, Lin LC, Wang KL and Hsia SM: Isoliquiritigenin induces apoptosis and autophagy and inhibits endometrial cancer growth in mice. Oncotarget 7: 73432-73447, 2016.

23. Li Y, Zhao H, Wang Y, Zheng H, Yu W, Chai H, Zhang J, Falck JR, Guo AM, Yue J, et al: Isoliquiritigenin induces growth inhibition and apoptosis through downregulating arachidonic acid metabolic network and the deactivation of PI3K/Akt in human breast cancer. Toxicol Appl Pharmacol 272: 37-48, 2013.

24. Wu S, Xue J, Yang Y, Zhu H, Chen F, Wang J, Lou G, Liu Y, Shi Y, Yu Y, et al: Isoliquiritigenin inhibits interferon- $\gamma$-inducible genes expression in hepatocytes through down-regulating activation of JAK1/STAT1, IRF3/MyD88, ERK/MAPK, JNK/MAPK and PI3K/Akt signaling pathways. Cell Physiol Biochem 37: 501-514, 2015.

25. Xu G, Zhang W, Bertram P, Zheng XF and Mcleod H: Pharmacogenomic profiling of the PI3K/PTEN-AKT-mTOR pathway in common human tumors. Int J Oncol 24: 893-900, 2004.

26. Zhou Y and Ho WS: Combination of liquiritin, isoliquiritin and isoliquirigenin induce apoptotic cell death through upregulating p53 and p21 in the A549 non-small cell lung cancer cells. Oncol Rep 31: 298-304, 2014. 
27. Xiao $\mathrm{C}, \mathrm{Wu} \mathrm{CH}$ and $\mathrm{Hu} \mathrm{HZ}$ : LncRNA UCA1 promotes epithelial-mesenchymal transition (EMT) of breast cancer cells via enhancing Wnt/beta-catenin signaling pathway. Eur Rev Med Pharmacol Sci 20: 2819-2824, 2016.

28. Magnaldo T and Sarasin A: Xeroderma pigmentosum: From symptoms and genetics to gene-based skin therapy. Cells Tissues Organs 177: 189-198, 2004.

29. Meier P, Finch A and Evan G: Apoptosis in development. Nature 407: 796-801, 2000

30. Kerr JF, Wyllie AH and Currie AR: Apoptosis: A basic biological phenomenon with wide-ranging implications in tissue kinetics. Br J Cancer 26: 239-257, 1972

31. Ola MS, Nawaz M and Ahsan H: Role of Bcl-2 family proteins and caspases in the regulation of apoptosis. Mol Cell Biochem 351: 41-58, 2011.

32. Borner C: The Bcl-2 protein family: Sensors and checkpoints for life-or-death decisions. Mol Immunol 39: 615-647, 2003.

33. Brown R: The bcl-2 family of proteins. Br Med Bull 53: 466-477, 1997.

34. Zhao H, Yenari MA, Cheng D, Sapolsky RM and Steinberg GK: Bcl-2 overexpression protects against neuron loss within the ischemic margin following experimental stroke and inhibits cytochrome c translocation and caspase-3 activity. J Neurochem 85 1026-1036, 2003.
35. Zhou GS, Song LJ and Yang B: Isoliquiritigenin inhibits proliferation and induces apoptosis of U87 human glioma cells in vitro. Mol Med Rep 7: 531-536, 2013.

36. Jung JI, Chung E, Mi RS, Seon MR, Shin HK, Kim EJ, Lim SS, Chung WY, Park KK and Park JH: Isoliquiritigenin (ISL) inhibits ErbB3 signaling in prostate cancer cells. Biofactors 28: $159-168,2006$

37. Kang SW, Choi JS, Choi YJ, Bae JY, Li J, Kim DS, Kim JL, Shin SY, Lee YJ, Kwun IS and Kang YH: Licorice isoliquiritigenin dampens angiogenic activity via inhibition of MAPK-responsive signaling pathways leading to induction of matrix metalloproteinases. J Nutr Biochem 21: $55-65,2010$.

38. Kim N, Jeong S, Jing K, Shin S, Kim S, Heo JY, Kweon GR, Park SK, Wu T, Park JI and Lim K: Docosahexaenoic acid induces cell death in human non-small cell lung cancer cells by repressing mTOR via AMPK activation and PI3K/akt inhibition. Biomed Res Int 2015: 239764, 2015

39. Li YC, He SM, He ZX, Li M, Yang Y, Pang JX, Zhang X, Chow K, Zhou Q, Duan W, et al: Plumbagin induces apoptotic and autophagic cell death through inhibition of the PI3K/Akt/mTOR pathway in human non-small cell lung cancer cells. Cancer Lett 344: 239-259, 2014. 